\title{
NR3C2 Gene is Associated with Susceptibility to High-Altitude Pulmonary Edema in Han Chinese
}

\author{
Yingzhong Yang, MD, PhD; Jin Xu, PhD; Feng Tang, PhD; Qin Ga, MD; Yuhong Li, MD, PhD; \\ Wei Guan, MD, PhD; Ri-Li Ge, MD, PhD \\ From the Research Center for High Altitude Medical Sciences, School of Medicine, Qinghai University, Qinghai, China (Drs Yang, Tang, Ga, and \\ Ge); the Basic and Applied Key Laboratory for High Altitude Medical Science and Technology of Qinghai, Qinghai, China (Drs Yang, Tang, Ga, \\ and Ge); the Qinghai-Utah United Key Laboratory for High Altitude Medical Science, Qinghai, China (Drs Yang, Tang, Ga, and Ge); the Basic \\ Medical Sciences, School of Medicine, Qinghai University, Qinghai, China (Dr Xu); and the Department of Respiration, Affiliated Hospital, \\ Qinghai University, Qinghai, China (Drs Li and Guan).
}

\begin{abstract}
Introduction-The mineralocorticoid receptor is encoded by the $N R 3 C 2$ gene and plays an important role in regulating vascular tone in high-altitude pulmonary edema (HAPE). This study aimed to investigate the association of the polymorphisms in the NR3C2 gene with HAPE susceptibility in Han Chinese.

Methods-We enrolled 133 HAPE patients and 131 matched healthy Han Chinese from the Yushu area in Qinghai, where the altitude is greater than $3500 \mathrm{~m}$. Two single nucleotide polymorphisms (SNPs) of the NR3C2 gene, rs2070951 and rs5522, were genotyped by the Sequenom MassARRAY SNP assay.

Results-The genotypic distributions and allele frequencies of NR3C2 SNP rs5522 were significantly different between the HAPE and control groups $(P<0.05)$. The frequency of the A allele of rs5522 was significantly higher in the HAPE group than in the control group $(P<0.05)$ with an odds ratio of 1.7 (95\% CI: $1.0-2.8$ ). There were no significant differences in the genotypic distributions and allele frequencies of NR3C2 SNP rs2070951 between the HAPE and control groups. The frequencies of the C-A and C-G haplotypes were significantly higher in the HAPE group than in control group.

Conclusions - The rs5522 polymorphism of the NR3C2 gene was associated with HAPE susceptibility in Chinese subjects. The A allele may contribute to the susceptibility to HAPE. The frequency of the C-A and C-G haplotypes of rs2070951 and rs5522 in the NR3C2 gene may increase the risk of HAPE.
\end{abstract}

Keywords: polymorphism, rs2070951, rs5522, genotype, genetic, HAPE

\section{Introduction}

High-altitude pulmonary edema (HAPE) usually occurs at altitudes above $3000 \mathrm{~m}$ in rapidly ascending nonacclimatized individuals within the first week after arrival. ${ }^{1,2}$ The initial pathogenesis is due to nonuniform pulmonary vasoconstriction and pulmonary capillary stress failure, with edema in pulmonary interstitial tissue and alveoli, leading to a high permeability type of edema. ${ }^{3}$ Although hypoxia is a major trigger factor, the pathogenesis of HAPE remains unclear because some individuals are more susceptible to HAPE upon exposure

Corresponding author: Yingzhong Yang, MD, PhD, Research Center for High Altitude Medical Sciences, Qinghai University School of Medicine, 16 Kunlun Rd, Xining 810001, Qinghai, PR CHINA; e-mail: yingzhong-yang@hotmail.com.

Submitted for publication November 2017.

Accepted for publication July 2018. to the same hypoxia conditions. HAPE is considered a multifactorial condition that involves both genetic and environmental factors. ${ }^{4}$ Growing evidence suggests that genetic factors play an important role in the risk of HAPE. ${ }^{5-7}$ However, the exact genetic mechanism behind its pathogenesis is largely unknown.

Exposure to stress results in a spectrum of responses. In response to stress the hypothalamic-pituitaryadrenal axis is activated to cause a transient increase in circulating cortical hormone, which exerts effects through the glucocorticoid receptor and mineralocorticoid receptor (MR). Aldosterone, the ligand of MR, is one product of the renin-angiotensin-aldosterone system, which plays a key role in the control of sodium and potassium balance in the kidney and body water content and blood pressure. Normally, activation of MR by the steroid hormone aldosterone raises renal salt reabsorption by increasing activity of the epithelial sodium 
channel of the distal nephron. ${ }^{6}$ MR mediates sympathetic regulation of blood volume homeostasis and influences salt appetite. ${ }^{8}$ The relationship between aldosterone and the risk of HAPE is unclear, with some studies reporting an increase in serum aldosterone in highland travelers and other studies reporting a decrease. ${ }^{9,10}$ Lower levels of aldosterone are a selective advantage at high altitude. ${ }^{11}$ Therefore, MR may play an important role in regulating vascular tone in HAPE. ${ }^{12}$

MR is encoded by the $N R 3 C 2$ gene located at $4 \mathrm{q} 31$, and the gene contains 10 exons. ${ }^{13}$ The first 2 exons $1 \alpha$ and $1 \beta$ are untranslated and result in 2 different mRNA isoforms. Exons 3 and 4 encode the DNA binding domain, exons 5-8 and the first part of exon 9 encode the ligand binding domain. ${ }^{14}$ MR has been extensively studied and found to play a role not only in the kidney but also in extrarenal tissues. Several studies confirmed expression of MR in human pulmonary artery endothelial cells and smooth muscle cells. ${ }^{15}$ MR inhibition prevents the progression of monocrotaline-induced pulmonary arterial hypertension in rats, and MR activation induces smooth muscle cell proliferation, which may contribute to pulmonary arterial hypertension. It has also been demonstrated that aldosterone induces a sulfenic posttranslational modification of the endothelin receptor type $\mathrm{B}$, leading to reduced nitric oxide bioavailability in the pulmonary artery. ${ }^{16} \mathrm{MR}$ regulates genes involved in vascular fibrosis, inflammation, and calcification and contributes to coronary endothelial cells inflammation and oxidative stress. ${ }^{17}$

Genetic variants in the $N R 3 C 2$ gene change renal salt reabsorption, alter blood pressure, and are correlated with pregnancy-related hypertension. ${ }^{18}$ The $215 \mathrm{G} / \mathrm{C}$ (rs2070951) and I180V (rs5522) SNPs in the NR3C2 gene have been associated with differential response to cortisol in vitro and in vivo. ${ }^{14}$ SNPs rs2070951 and rs5522, located 2 nucleotides before the translation site of exon 2 and within exon 2, respectively, have been demonstrated to affect in vitro transactivation by altering either MR expression or functionality. The rs5522 SNP substitutes isoleucine (A) for valine $(G)$ in the $\mathrm{N}$-terminal of MR protein and results in a mild loss of function compared with the isoallele. The rs5522 SNP has been associated with 1) heightened endocrine and autonomic responses to acute stress; 2) diminished cortisol-induced MR gene expression; and 3) geriatric depressive symptoms. ${ }^{19}$ The rs2070951 SNP is located outside the NR3C2 coding region but inside a Kozak translation regulatory sequence, which regulates MR transcription. In vitro, the $\mathrm{C}$-allele results in increased MR expression. ${ }^{14}$

In this study we tested the hypothesis that the 2 functional polymorphisms, rs2070951 and rs5522, in the $N R 3 C 2$ gene are associated with susceptibility to HAPE in Han Chinese.

\section{Methods \\ SUBJECTS}

In total, 133 Han Chinese who were admitted to Yushu People's Hospital between March 2010 and June 2015 for onset of HAPE 1 to 7 days after arrival at Yushu (3760 m) were recruited in this study as HAPE patients (HAPE-p) and included in final analysis. The diagnosis of HAPE was based on chest radiographs and standard diagnostic criteria. ${ }^{20}$ In total, 131 healthy controls (resistant to HAPE, HAPE-r) were randomly selected from the coworkers of HAPE-p individuals, matching the patients in age, sex, ethnicity, and working conditions (Table 1). These subjects remained healthy after working at Yushu for at least $3 \mathrm{mo}$, without experiencing HAPE or high-altitude cerebral edema. Because they were coworkers, all subjects from both groups came from the same elevation and had same ascent profile and working conditions.

The study protocols were approved by the Ethics Committee of Qinghai University (Xining, China). All participants in this study signed informed consent.

\section{MEASUREMENTS}

Hemoglobin concentration, hematocrit, percent oxygen saturation $\left(\mathrm{S}_{\mathrm{p}} \mathrm{O}_{2}\right)$, and heart rate were measured using

Table 1. High-altitude exposures and physiological phenotypes for the study populations

\begin{tabular}{lccccccc}
\hline Group & Number & Sex & Age $(y)$ & HGB $\left(g \cdot d L^{-1}\right)$ & Hct $(\%)$ & HR $\left(\right.$ beats $\left.\cdot \mathrm{min}^{-1}\right)$ & $S_{p} O_{2}(\%)$ \\
\hline HAPE-p & 133 & Male & $40.2 \pm 9.9$ & $157.2 \pm 15.2^{a}$ & $47.8 \pm 5.0^{a}$ & $110 \pm 15^{a}$ & $62.5 \pm 11.9^{a}$ \\
HAPE-r & 131 & Male & $40.4 \pm 4.7$ & $173.0 \pm 14.2$ & $51.2 \pm 7.2$ & $81 \pm 12$ & $88.9 \pm 4.2$ \\
\hline
\end{tabular}

HGB, hemoglobin; Hct, hematocrit; HR, heart rate; $\mathrm{S}_{\mathrm{p}} \mathrm{O}_{2}$, oxyhemoglobin saturation; HAPE-p, high altitude pulmonary edema patients; HAPE-r, high altitude pulmonary edema resistant (control).

Values were mean \pm SD.

${ }^{a} P<0.05$ vs HAPE-r. 
the Mindray Hematology Analyzer (BC-2300, Shenzhen, China) and a pulse oximeter (Ohmeda 3700 Pulse Oximeter; Datex-Ohmeda, Boulder, CO). These parameters were measured in the HAPE-p group on the first day of their stay at hospital and in the HAPE-r group once after having worked at Yushu for at least 3 mo. Data are presented as mean $\pm \mathrm{SD}$.

\section{DNA EXTRACTION AND GENOTYPING}

Five milliliters of venous blood samples were collected from each participant. The whole blood was separated into blood cells and plasma by centrifuging at $1000 \mathrm{~g}$ for $10 \mathrm{~min}$, and then the blood cells and plasma were transported to Xining for biochemical assays and genetic polymorphism analysis. Genomic DNA was extracted from venous blood by Gentra Puregene Blood Kit (Qiagen, 158389, Hilden, Germany) according to standard procedures. rs2070951 and rs5522 were genotyped by the single base extension detecting technology (iPLEX) (Capital Bio Corporation, Beijing, China). SNP loci-tested polymerase chain reaction (PCR) primers and single-base extension primers were designed by using the Sequenom MassARRAY Assay Design Genotyping Software and Tools (Sequenom, San Diego, CA). The PCR reaction consisted of $4.0 \mu \mathrm{L}$ of PCR Master Mixture (Takara Bio Inc, Kusatsu, Japan), $1 \mu \mathrm{L}$ of primers mixture, $1.0 \mu \mathrm{L}$ of genomic DNA (gDNA), $1.9 \mu \mathrm{L}$ of $\mathrm{ddH}_{2} \mathrm{O}$, and $0.1 \mu \mathrm{L}$ of deoxynucleotide (dNTPs). PCR was performed under the following thermal cycling conditions: $94^{\circ} \mathrm{C}$ for $4 \mathrm{~min}$; then $94^{\circ} \mathrm{C}$ for $20 \mathrm{~s}, 56^{\circ} \mathrm{C}$ for $30 \mathrm{~s}$, and $72^{\circ} \mathrm{C}$ for $1 \mathrm{~min}$ for 45 cycles; and finally $72^{\circ} \mathrm{C}$ for $4 \mathrm{~min}$. PCR products were treated with shrimp alkaline phosphatase to remove free deoxyribonucleoside triphosphates, and single-base extension reaction was performed in $2.0 \mu \mathrm{L}$ of EXTEND MIX, $0.619 \mu \mathrm{L}$ of $\mathrm{ddH}_{2} \mathrm{O}$, $0.94 \mu \mathrm{L}$ of Extend primer mix, $0.2 \mu \mathrm{L}$ of iPLEX buffer plus, $0.2 \mu \mathrm{L}$ of iPLEX terminator, and $0.04 \mu \mathrm{L}$ of iPLEX enzyme (Sequenom, San Diego, CA). The thermal cycling conditions were as follows: $94^{\circ} \mathrm{C}$ for $30 \mathrm{~s}$; then $94^{\circ} \mathrm{C}$ for $5 \mathrm{~s}, 52^{\circ} \mathrm{C}$ for $5 \mathrm{~s}$, and $80^{\circ} \mathrm{C}$ for $5 \mathrm{~s}$ for 40 cycles; and finally $72^{\circ} \mathrm{C}$ for $3 \mathrm{~min}$. The MassARRAY Nanodispenser RS1000 (Capital Bio Corporation, Beijing, China) was used to dispense the purified extension products onto a 384-element SpectroCHIP bioarray (Sequenom, San Diego, CA), and mass spectrometric analysis was performed using the MALDI-TOF (matrix-assisted laser desorption/ionization-time of flight) (Sequenom, San Diego, CA). The results were analyzed using TYPER 4.0 software (Sequenom, San Diego, CA).

\section{STATISTICAL ANALYSIS}

SPSS software (version 17.0; SPSS Inc, Chicago, IL) was used for statistical analysis. Deviations from HardyWeinberg equilibrium were assessed by the $\chi^{2}$ test for genotype frequency. Allele frequencies were calculated based on genotype frequencies in HAPE and control groups, and the intergroup difference was estimated with the $\chi^{2}$ test. $P<0.005$ was considered significant. Haplotype frequencies and the expected number of haplotypes for each individual were performed using SHEsis online software (http://analysis.bio-X.cn).

\section{Results}

The age, heart rate, $\mathrm{S}_{\mathrm{p}} \mathrm{O}_{2}$, and concentration of hemoglobin and hematocrit for the HAPE-p and HAPE-r groups are listed in Table 1 . We found that $\mathrm{S}_{\mathrm{p}} \mathrm{O}_{2}$ was significantly lower and heart rate was significantly higher in the HAPE-p group compared with the HAPE-r group.

The genotypic distributions and allelic frequencies of NR3C2 SNPs (rs2070951 and rs5522) were in HardyWeinberg equilibrium in HAPE-p and HAPE-r groups. For rs2070951, the genotype frequencies for CC, CG, GG in HAPE-p and HAPE-r groups were $58.7 \%, 36.1 \%$, $5.2 \%$ and $52.7 \%, 38.9 \%, 8.4 \%$, respectively. The allelic frequencies of $\mathrm{C}$ and $\mathrm{G}$ were $51.9 \%, 45.9 \%$ and $48.1 \%$, $54.1 \%$, respectively. There were no significant differences in the genotypic and allelic frequencies between the 2 groups (Table 2 ).

For rs5522, the genotype frequencies for AA, AG, GG in HAPE-p and HAPE-r groups were $76.7 \%, 23.3 \%, 0 \%$ and $65.7 \%, 32.1 \%, 2.3 \%$, respectively. The allelic frequencies of $\mathrm{A}$ and $\mathrm{G}$ were $52.3 \%, 39.2 \%$ and $47.7 \%$, $60.8 \%$, respectively. The A allele was significantly more prevalent among the HAPE-p group than the HAPE-r group $(P<0.05)$ with an odds ratio of $1.7(95 \%$ CI: 1.0-2.8). There were significant differences in the genotypic distributions and allelic frequencies between the 2 groups $(P<0.05)$ (Table 3$)$.

Based on our data, we constructed haplotypes in the order of rs2070951 followed by rs5522 (Table 4). The results indicate that the frequencies of the C-A and C-G haplotypes were significantly higher in the HAPE-p group than in the HAPE-r group, with the odds ratio of $1.6(1.1-2.3)$ $(P=0.009)$ and $0.6(0.4-1.0)(P=0.03)$, respectively.

\section{Discussion}

We found no significant difference in the genotypic distributions and allele frequencies of rs2070951 between HAPE-susceptible and HAPE-resistant individuals. In contrast, we did identify significant differences in rs5522 
Table 2. Genotypic distributions and allelic frequencies of the rs2070951 in 2 groups

\begin{tabular}{llll}
\hline Variables & HAPE- $p(n \%)$ & HAPE-r $(n \%)$ & OR (95\% CI) \\
\hline Genotypic frequency & & & \\
CC & $78(58.7 \%)$ & $69(52.7 \%)$ & $1.2(0.7-2.0)$ \\
CG & $48(36.1 \%)$ & $51(38.9 \%)$ & $1.776(0.7-4.8)$ \\
GG & $7(5.2 \%)$ & $11(8.4 \%)$ & \\
Allelic positivity & $\chi^{2}=1.5$ & $P=0.469$ & \\
$\quad$ CC positivity (CC plus CG) & $126(51.2 \%)$ & $120(48.8 \%)$ & $1.2(0.8-1.8)$ \\
GG positivity (GG plus CG) & $55(47.0 \%)$ & $62(53.0 \%)$ & \\
Allelic frequency & $204(76.7 \%)$ & $189(72.1 \%)$ & \\
C & $62(23.3 \%)$ & $73(27.9 \%)$ & $1.3(0.9-1.9)$ \\
G & & & \\
\hline
\end{tabular}

HAPE-p, high altitude pulmonary edema patients; HAPE-r, high altitude pulmonary edema resistant (control); OR, odds ratio.

Table 3. Genotypic distributions and allelic frequencies of the rs5522 in 2 groups

\begin{tabular}{|c|c|c|c|c|}
\hline Variables & $H A P E-p(n \%)$ & $H A P E-r(n \%)$ & OR $(95 \% C I)$ & $P$ value \\
\hline \multicolumn{5}{|l|}{ Genotypic frequency } \\
\hline AA & $102(76.7 \%)$ & $86(65.6 \%)$ & & \\
\hline $\mathrm{AG}$ & $31(23.3 \%)$ & $42(32.1 \%)$ & $1.6(0.9-2.8)$ & 0.087 \\
\hline \multirow[t]{2}{*}{ GG } & 0 & $3(2.3 \%)$ & $0.5(0.4-0.5)$ & 0.062 \\
\hline & $\chi^{2}=6.004$ & $P=0.05$ & & \\
\hline \multicolumn{5}{|l|}{ Allelic positivity } \\
\hline AA positivity (AA plus AG) & $133(51.0 \%)$ & $128(49.0 \%)$ & & \\
\hline GG positivity (GG plus AG) & $31(40.8 \%)$ & $45(59.2 \%)$ & $1.5(0.9-2.5)$ & 0.119 \\
\hline \multicolumn{5}{|l|}{ Allelic frequency } \\
\hline A & $235(88.3 \%)$ & $214(81.7 \%)$ & & \\
\hline G & $31(11.7 \%)$ & $48(18.3 \%)$ & $1.7(1.0-2.8)$ & 0.032 \\
\hline
\end{tabular}

HAPE-p, high altitude pulmonary edema patients; HAPE-r, high altitude pulmonary edema resistant (control); OR, odds ratio.

Table 4. Haplotype analysis for the rs2070951 and rs5522 in 2 groups

\begin{tabular}{llllll}
\hline Haplotype & HAPE- $p(n \%)$ & HAPE-r $(n \%)$ & $\chi^{2}$ & OR $(95 \%$ CI $)$ & P value \\
\hline $\mathrm{C}-\mathrm{A}^{a}$ & $173(65.0 \%)$ & $141(53.8 \%)$ & 6.895 & $1.6(1.1-2.3)$ & 0.009 \\
$\mathrm{C}-G^{a}$ & $31(11.7 \%)$ & $48(18.3 \%)$ & 4.610 & $0.6(0.4-1.0)$ & 0.032 \\
$\mathrm{G}-\mathrm{A}$ & $62(23.3 \%)$ & $73(27.9 \%)$ & 1.439 & $0.8(0.5-1.2)$ & 0.230 \\
\hline
\end{tabular}

HAPE-p, high altitude pulmonary edema patients; HAPE-r, high altitude pulmonary edema resistant (control); OR, odds ratio.

${ }^{a}$ Statistical significance after controlling for multiple comparisons.

between these groups. The A allele was significantly more prevalent among the HAPE-p group than among the HAPE-r group $(P<0.05)$. We speculated that allelic $\mathrm{A}$ is prevalent among the HAPE-p group and thus aldosterone may have greater effect on the HAPE-p group than on the HAPE-r group. In contrast, allelic $\mathrm{G}$ is prevalent among the HAPE-r group, and the Val-allele results in a mild loss of function of MR. Further studies are needed to investigate the effect of MR variability on aldosterone function in vivo at high altitude. Our study also indicates that the frequency of the C-A and C-G haplotypes of the NR3C2 gene may increase the risk of HAPE.

Several limitations of this study should be pointed out. First, we only genotyped 2 SNPs in the $N R 3 C 2$ gene in this study. Other SNPs in the NR3C2 gene should be examined in our future studies. Second, our study enrolled only a moderate number of subjects; we would recommend a larger scale investigation to confirm our findings. 
Acknowledgments: We thank Dr Jiangxi from the Yushu People's Hospital and Dr Nima from the Yushu Bayi Hospital for sample collection.

Author Contributions: Study design and concept (YY); statistical analysis (JX); sample collection (FT, QG, YL, WG, RG).

Financial/Material Support: This work was supported by the Natural Science Foundation of China (No. 81760335), Qinghai Thousand Talents Program, The second round of "Qinghai 135 high-level talents training project," and the Science and Technology Support Program of Qinghai (2015-SF-124).

Disclosures: None.

\section{References}

1. Hackett PH, Roach RC. High-altitude illness. N Engl J Med. 2001;345(2):107-14.

2. Basnyat B, Murdoch DR. High-altitude illness. Lancet. 2003;361(9373):1967-74.

3. Maggiorini M. High altitude-induced pulmonary oedema. Cardiovasc Res. 2006;72(1):41-50.

4. Yang YZ, Wang YP, Qi YJ, Du Y, Ma L, Ga Q, et al. Endothelial PAS Domain Protein 1 Chr2:46441523(hg18) Polymorphism is associated with susceptibility to high sltitude pulmonary edema in Han Chinese. Wilderness Environ Med. 2013;24(4):315-20.

5. Mortimer H, Patel S, Peacock AJ. The genetic basis of high-altitude pulmonary oedema. Pharmacol Ther. 2004;101(2):183-92.

6. Fortier MÈ, Sengupta SM, Grizenko N, Choudhry Z, Thakur G, Joober R. Genetic evidence for the association of the hypothalamic-pituitary-adrenal (HPA) axis with ADHD and methylphenidate treatment response. Neuromolecular Med. 2013;15(1):122-32.

7. MacInnis MJ, Koehle MS. Evidence for and against genetic predispositions to acute and chronic altitude illnesses. High Altitude Med Biol. 2016;17(4):281-93.

8. de Kloet ER, Van Acker SA, Sibug RM, Oitzl MS, Meijer OC, Rahmouni K, et al. Brain mineralocorticoid receptors and centrally regulated functions. Kidney Int. 2000;57:1329-36.

9. Li YY, Luo DC, Xiao Q. Clinical significance of changes in plasma renin-angiotensin aldosterone system in patients with high altitude pulmonary edema [in Chinese]. Zhonghua Nei Ke Za Zhi. 1993;32(4):232-4.

10. Zaccaria M, Rocco S, Noventa D, Varnier M, Opocher G. Sodium regulating hormones at high altitude: basal and post-exercise levels. J Clin Endocrinol Metab. 1998;83 (2): $570-4$

11. Rajput C, Arif E, Vibhuti A, Stobdan T, Khan AP, Norboo $\mathrm{T}$, et al. Predominance of interaction among wild-type alleles of CYP11B2 in Himalayan natives associates with high-altitude adaptation. Biochem Biophys Res Commun. 2006;348(2):735-40.

12. Srivastava S, Bhagi S, Kumari B, Chandra K, Sarkar S, Ashraf MZ. Association of polymorphisms in angiotensin and aldosterone synthase genes of the renin-angiotensinaldosterone system with high-altitude pulmonary edema. J Renin Angiotensin Aldosterone Syst. 2012;13(1):155-60.

13. Arriza JL, Weinberger C, Cerelli G, Glaser TM, Handelin BL, Housman DE, et al. Cloning of human mineralocorticoid receptor complementary DNA: structural and functional kinship with the glucocorticoid receptor. Science. 1987;237(4812):268-75.

14. ter Heegde F, De Rijk RH, Vinkers CH. The brain mineralocorticoid receptor and stress resilience. Psychoneuroendocrinology. 2015;52:92-110.

15. Hatakeyama H, Miyamori I, Fujita T, Takeda Y, Takeda R, Yamamoto H. Vascular aldosterone. Biosynthesis and a link to angiotensin II-induced hypertrophy of vascular smooth muscle cells. J Biol Chem. 1994;269(39):24316-20.

16. Maron BA, Zhang YY, White K, Chan SY, Handy DE, Mahoney CE, et al. Aldosterone inactivates the endothelin-B receptor via a cysteinyl thiol redox switch to decrease pulmonary endothelial nitric oxide levels and modulate pulmonary arterial hypertension. Circulation. 2012;126(8):963-74.

17. Dupont JJ, Jaffe IZ. 30 years of the mineralocorticoid receptor: the role of the mineralocorticoid receptor in the vasculature. J Endocrinol. 2017;234(1):T67-82.

18. DeRijk RH, Wüst S, Meijer OC, Zennaro MC, Federenko IS, Hellhammer DH, et al. A common polymorphism in the mineralocorticoid receptor modulates stress responsiveness. J Clin Endocrinol Metab. 2006;91(12):5083-9.

19. Bogdan R, Perlis RH, Fagerness J, Pizzagalli DA. The impact of mineralocorticoid receptor ISO/VAL genotype (rs5522) and stress on reward learning. Genes Brain Behav. 2010;9(6):658-67.

20. Gabry AL, Ledoux X, Mozziconacci M, Martin C. Highaltitude pulmonary edema at moderate altitude $(<2,400 \mathrm{~m}$; 7,870 feet): a series of 52 patients. Chest. 2003;123(1):49-53. 